\title{
Regularity Result for Quasilinear Elliptic Systems with Super Quadratic Natural Growth Condition
}

\author{
Shuhong Chen ${ }^{1}$ and Zhong Tan ${ }^{2}$ \\ ${ }^{1}$ Department of Mathematics and Information Science, Zhangzhou Normal University, Zhangzhou, Fujian 363000, China \\ ${ }^{2}$ School of Mathematical Science, Xiamen University, Xiamen, Fujian 361005, China
}

Correspondence should be addressed to Shuhong Chen; shiny0320@163.com

Received 31 December 2012; Accepted 2 April 2013

Academic Editor: Paul Eloe

Copyright (C) 2013 S. Chen and Z. Tan. This is an open access article distributed under the Creative Commons Attribution License, which permits unrestricted use, distribution, and reproduction in any medium, provided the original work is properly cited.

We consider boundary regularity for weak solutions of second-order quasilinear elliptic systems under natural growth condition with super quadratic growth and obtain a general criterion for a weak solution to be regular in the neighborhood of a given boundary point. Combined with existing results on interior partial regularity, this result yields an upper bound on the Hausdorff dimension of the singular set at the boundary.

\section{Introduction}

This paper considers boundary regularity for weak solutions of quasilinear elliptic systems

$$
-D_{\alpha}\left(A_{i j}^{\alpha \beta}(x, u) D_{\beta} u^{j}\right)=B_{i}(x, u, D u), \quad x \in \Omega,
$$

where $\Omega$ is a bounded domain in $R^{n}$ with boundary of class $C^{1}, n \geq 2$ and $u$ takes value in $R^{N}, N>1$. Each $A_{i j}^{\alpha \beta}$ maps $\Omega \times R^{N}$ into $R$, and each $B_{i}$ maps $\Omega \times R^{N} \times R^{n N}$ into $R$. A partial regularity theory of (1) must have a priori existence weak solutions. Here we assume that weak solutions exist and consider partial regularity of weak solutions directly. We further impose certain structural conditions on $A_{i j}^{\alpha \beta}$ and $B_{i}$ with $m>2$ as follows.

(H1) There exists $L>0$ such that

$$
\begin{gathered}
A_{i j}^{\alpha \beta}(x, \xi)(\nu, \widetilde{\nu}) \leq L\left(1+|\xi|^{2}\right)^{(m-2) / 2}|\nu||\widetilde{\nu}| \\
\quad \text { for all }(x, \xi) \in \bar{\Omega} \times R^{N}, \nu, \widetilde{\nu} \in R^{n N} .
\end{gathered}
$$

(H2) $A_{i j}^{\alpha \beta}(x, \xi)$ is uniformly strongly elliptic; that is, for some $\lambda>0$ we have

$$
\begin{gathered}
A_{i j}^{\alpha \beta}(x, \xi)(\nu, \nu) \geq \lambda\left(1+|\xi|^{2}\right)^{(m-2) / 2}|\nu|^{2} \\
\quad \text { for all }(x, \xi) \in \bar{\Omega} \times R^{N}, \nu \in R^{n N} .
\end{gathered}
$$

(H3) Assume that $A_{i j}^{\alpha \beta} \in C^{0}\left(\Omega \times R^{N}, R^{n N}\right)$ and further that $A_{i j}^{\alpha \beta}$ is uniformly continuous on sets of the form $\bar{\Omega} \times$ $\{\xi:|\xi| \leq M\}$, for any fixed $M, 0<M<\infty$.

(H4) (Natural growth condition). There exist constants $a$ and $b$, with $a$ possibly depending on $M>0$, such that

$$
\left|B_{i}(x, \xi, \nu)\right| \leq a(M)|\nu|^{m}+b
$$

for all $x \in \bar{\Omega}, \xi \in R^{N}$ with $|\xi| \leq M$ and $\nu \in R^{n N}$.

Further hypothesis (H3) deduces, writing $\omega(\cdot)$ for $\omega(M, \cdot)$, the existence of a monotone nondecreasing concave function $\omega:[0, \infty) \rightarrow[0, \infty)$ with $\omega(0)=0$, continuous at 0 , such that

$$
\left|A_{i j}^{\alpha \beta}(x, u)-A_{i j}^{\alpha \beta}(y, v)\right| \leq \omega\left(|x-y|^{m}+|u-v|^{m}\right),
$$

for all $x, y \in \bar{\Omega}, u, v \in R^{N}$ with $|u|,|v| \leq M[1]$. 
(H5) There exist $s$ with $s>n$ and a function $g \in H^{1, s}(\Omega$, $R^{N}$ ), such that

$$
\left.u\right|_{\partial \Omega}=\left.g\right|_{\partial \Omega} .
$$

Note that we trivially have $g \in H^{1,2}\left(\Omega, R^{N}\right)$. Further, by the Sobolev embedding theorem we have $g \in C^{0, \kappa}\left(\Omega, R^{N}\right)$ for any $\kappa \in[0,1-(n / s)]$. If $\left.g\right|_{\partial \Omega} \equiv 0$, we will take $g \equiv 0$ on $\Omega$.

If the domain we consider is an upper half unit ball $B^{+}$, the boundary condition becomes as follows.

(H5)' There exist $s$ with $s>n$ and a function $g \in$ $H^{1, s}\left(B^{+}, R^{N}\right)$, such that

$$
\left.u\right|_{D}=\left.g\right|_{D} .
$$

Here we write $B_{\rho}\left(x_{0}\right)=\left\{x \in R^{n}:\left|x-x_{0}\right|<\rho\right\}$, and further $B_{\rho}=B_{\rho}(0), B=B_{1}$. Similarly we denote upper half balls as follows: for $x_{0} \in R^{n-1} \times\{0\}$, we write $B_{\rho}^{+}\left(x_{0}\right)$ for $\{x \in$ $\left.R^{n}: x_{n}>0,\left|x-x_{0}\right|<\rho\right\}$ and set $B_{\rho}^{+}=B_{\rho}^{+}(0), B^{+}=B_{1}^{+}$. For $x_{0} \in R^{n-1} \times\{0\}$ we further write $D_{\rho}\left(x_{0}\right)$ for $\left\{x \in R^{n}: x_{n}=\right.$ $\left.0,\left|x-x_{0}\right|<\rho\right\}$ and set $D_{\rho}=D_{\rho}(0), D=D_{1}$.

Definition 1. By a weak solution of (1) one means a vector valued function $u \in W^{1, m}\left(\bar{\Omega}, R^{N}\right) \cap L^{\infty}\left(\bar{\Omega}, R^{N}\right)$ such that

$$
\int_{\Omega} A_{i j}^{\alpha \beta}(x, u)\left(D_{\beta} u^{j}, D_{\alpha} \varphi^{i}\right) d x=\int_{\Omega} B_{i}(x, u, D u) \cdot \varphi^{i} d x
$$

holds for all test-functions $\varphi \in C_{0}^{\infty}\left(\bar{\Omega}, R^{N}\right)$ and, by approximation, for all $\varphi \in W_{0}^{1, m}\left(\bar{\Omega}, R^{N}\right) \cap L^{\infty}\left(\bar{\Omega}, R^{N}\right)$.

Under such assumptions, even the boundary data is smooth, one cannot expect full regularity of (1) at the boundary [2]. Then, our goal is to establish partial boundary regularity.

After the partial regularity results of the type in this paper were proved by Giusti and Miranda in [3], there are some previous partial regularity results for quasilinear systems. For example, regularity up to boundary for nonlinear and quasilinear systems [4-6] has been studied by Arkhipova. Wiegner [7] established boundary regularity for systems in diagonal form first, and the proof was generalized and extended by Hildebrandt and Widman [8]. Jost and Meier [9] deduced full regularity in a neighborhood of the boundary for minima of functionals with the form $\int_{\Omega} A(x, u)|D u|^{2} d x$. Furthermore, Duzaar et al. obtained the boundary Hausdorff dimension on the singular sets of solutions to even more general systems in $[10,11]$ recently. Further discussion for regularity theory can be seen in $[12,13]$ and their references.

Inspired by [14], in this paper, we would establish boundary regularity for quasilinear systems under natural growth condition by the method of A-harmonic approximation.

The technique of A-harmonic approximation [15-17] is a natural extension of the harmonic approximation technique, which originated from Simon's proof of Allard's [18] $\varepsilon$ regularity theorem. In this context, using the A-harmonic approximation technique, we obtain the following regularity results.
Theorem 2. Consider a bounded domain $\Omega$ in $R^{N}$, with boundary of class $C^{1}$. Let $u$ be a bounded weak solution of (1) satisfying the boundary condition (H5), and $\|u\|_{L^{\infty}} \leq M<$ $\infty$ with $2 a(M) M<\lambda$, where the structure conditions (H1)(H3) hold for $A_{i j}^{\alpha \beta}$ and (H4) holds for $B_{i}$. Consider a fixed $\gamma \in(0, \sigma]$. Then there exist positive $R_{1}$ and $\varepsilon_{0}$ (depending only on $n, N, \lambda, L, b, M, a(M), \omega(\cdot), m$, and $\gamma)$ with the property that

$$
f_{B_{R}\left(x_{0}\right) \cap \Omega}\left|u-u_{x_{0}, R}^{\prime}\right|^{2} d x+\|g\|_{H^{1, s}}^{2} R^{2(1-(n / s))}+R^{2} \leq \varepsilon_{0}^{2}
$$

for some $R \in\left(0, R_{1}\right]$ for a given $x_{0} \in \partial \Omega$ implies $u \in$ $C^{0, \gamma}\left(\bar{B}_{R / 2}\left(x_{0}\right) \cap \bar{\Omega}, R^{N}\right)$.

Note in particular that the boundary condition (H5) means that $u_{x_{0}, R}^{\prime}$ makes sense: in fact, we have $u_{x_{0}, R}^{\prime}=g_{x_{0}, R}^{\prime}$. For $v \in L^{1}(\partial \Omega), x_{0} \in \partial \Omega$, we set $v_{x_{0}, R}^{\prime}=f_{\partial \Omega \cap \bar{B}_{R}\left(x_{0}\right)} \nu d H^{n-1}$. In particular, for $v \in L^{1}\left(D_{\rho}\left(x_{0}\right)\right), x_{0} \in D$, we write $v_{x_{0}, \rho}^{\prime}=$ $f_{D_{\rho}\left(x_{0}\right)} v d H^{n-1}$.

Combining this result with the analogous interior [19] and a standard covering argument allows us to obtain the following bound on the size of the singular set.

Corollary 3. Under the assumptions of Theorem 2 the singular set of the weak solution $u$ has $(n-2)$-dimensional Hausdorff measure zero in $\bar{\Omega}$.

If the domain of the main step in proving Theorem 2 is a half ball, the result then is the following.

Theorem 4. Consider a bounded weak solution of (1) on the upper half unit ball $B^{+}$which satisfies the boundary condition (H5)' and $\|u\|_{L^{\infty}} \leq M<\infty$ with $2 a(M) M<\lambda$, where the structure conditions (H1)-(H3) hold for $A_{i j}^{\alpha \beta}$ and (H4) holds for $B_{i}$. Then there exist positive $R_{0}$ and $\varepsilon_{0}$ (depending only on $n, N, \lambda, L, b, M, a(M), M, \omega(\cdot), m$, and $\gamma)$ with the property that

$$
f_{B_{R}^{+}\left(x_{0}\right)}\left|u-u_{x_{0}, R}^{\prime}\right|^{2} d x+\|g\|_{H^{1, s}}^{2} R^{2(1-(n / s))}+R^{2} \leq \varepsilon_{0}^{2},
$$

for some $R \in\left(0, R_{0}\right]$ for a given $x_{0} \in D$, implies that there holds: $u \in C^{0, \sigma}\left(\bar{B}_{R / 2}^{+}\left(x_{0}\right), R^{N}\right)$.

Note that analogous to the above, the boundary condition $(\mathrm{H} 5)^{\prime}$ ensures that $u_{x_{0}, R}^{\prime}$ exists, and we have indeed $u_{x_{0}, R}^{\prime}=$ $g_{x_{0}, R}^{\prime}$.

\section{The A-Harmonic Approximation Technique}

In this section we present the A-harmonic approximation lemma [14] and some standard results due to Companato [20]. 
Lemma 5 (A-harmonic approximation lemma). Consider fixed positive $\lambda$ and $L$, and $n, N \in N$ with $n \geq 2$. Then for any given $\varepsilon>0$ there exists $\delta=\delta(n, N, \lambda, L, \varepsilon) \in(0,1]$ with the following property: for any $A \in \operatorname{Bil}\left(R^{n N}\right)$ satisfying

$$
\begin{gathered}
A(\nu, \nu) \geq \lambda|\nu|^{2} \quad \text { for all } \nu \in R^{n N}, \\
|A(\nu, \bar{\nu})| \leq L|\nu||\bar{\nu}| \quad \text { for all } \nu, \bar{\nu} \in R^{n N}
\end{gathered}
$$

for any $w \in H^{1,2}\left(B_{\rho}^{+}\left(x_{0}\right), R^{N}\right)\left(\right.$ for some $\left.\rho>0, x_{0} \in R^{n}\right)$ satisfying

$$
\begin{gathered}
\rho^{2-n} \int_{B_{\rho}^{+}\left(x_{0}\right)}|D w|^{2} d x \leq 1, \\
\left|\rho^{2-n} \int_{B_{\rho}^{+}\left(x_{0}\right)} A(D w, D \varphi) d x\right| \leq \delta \rho \sup _{B_{\rho}^{+}\left(x_{0}\right)}|D \varphi|, \\
\left.w\right|_{D_{\rho}\left(x_{0}\right)}=0
\end{gathered}
$$

for all $\varphi \in C_{0}^{1}\left(B_{\rho}^{+}\left(x_{0}\right), R^{N}\right)$, there exists an A-harmonic function

$$
\begin{aligned}
v \in \widetilde{H}= & \left\{\widetilde{w} \in H^{1,2}\left(B_{\rho}^{+}\left(x_{0}\right), R^{N}\right)\right. \\
& \left.\left.\cdot\left|\rho^{2-n} \int_{B_{\rho}^{+}\left(x_{0}\right)}\right| D \widetilde{w}\right|^{2} d x \leq 1,\left.\widetilde{w}\right|_{D_{\rho}\left(x_{0}\right)} \equiv 0\right\}
\end{aligned}
$$

with

$$
\rho^{-n} \int_{B_{\rho}^{+}\left(x_{0}\right)}|v-w|^{2} d x \leq \varepsilon .
$$

Next we recall a slight modification of a characterization of Hölder continuous functions originally due to Campanato [21].

Lemma 6. Consider $n \in N, n \geq 2$, and $x_{0} \in R^{n-1} \times\{0\}$. Suppose that there are positive constants $\kappa$ and $\alpha$, with $\alpha \in$ $(0,1]$ such that, for some $v \in L^{2}\left(B_{6 R}^{+}\left(x_{0}\right)\right)$, there holds the following:

$$
\inf _{\mu \in R}\left\{\rho^{-n} \int_{B_{\rho}^{+}(y)}|\nu-\mu|^{2} d x\right\} \leq \kappa^{2}\left(\frac{\rho}{R}\right)^{2 \alpha},
$$

for all $y \in D_{2 R}\left(x_{0}\right)$ and $\rho \leq 4 R$; and

$$
\inf _{\mu \in R} \rho^{-n}\left\{\int_{B_{\rho}(y)}|\nu-\mu|^{2} d x\right\} \leq \kappa^{2}\left(\frac{\rho}{R}\right)^{2 \alpha},
$$

for all $y \in B_{2 R}^{+}\left(x_{0}\right)$ and $B_{\rho}(y) \subset B_{2 R}^{+}\left(x_{0}\right)$.

Then there exists a Hölder continuous representative of the $L^{2}$-class of $v$ on $\bar{B}_{R}^{+}\left(x_{0}\right)$, and for this representative $\bar{v}$ there holds

$$
|\bar{\nu}(x)-\bar{\nu}(z)| \leq C_{\kappa}\left(\frac{|x-z|}{R}\right)^{\alpha},
$$

for all $x, z \in \bar{B}_{R}^{+}\left(x_{0}\right)$, for a constant $C_{\kappa}$ depending only on $n$ and $\alpha$.
We close this section by a standard estimate for the solutions to homogeneous second-order elliptic systems with constant coefficients [20].

Lemma 7. Consider fixed positive $\lambda$ and $L$, and $n, N \in N$ with $n \geq 2$. Then there exists $C_{0}$ depending only on $n, N, \lambda$, and $L$ (without loss of generality we take $C_{0} \geq 1$ ) such that, for $A \in \operatorname{Bil}\left(R^{n N}\right)$ satisfying (11), any A-harmonic function $h$ on $B_{\rho}^{+}\left(x_{0}\right)$ with $\left.h\right|_{D_{\rho}\left(x_{0}\right)} \equiv 0$ satisfies

$$
\rho^{2} \sup _{B_{\rho / 2}^{+}\left(x_{0}\right)}|D h|^{2} \leq C_{0} \rho^{2-n} \int_{B_{\rho}^{+}\left(x_{0}\right)}|D h|^{2} d x .
$$

\section{The Caccioppoli Inequality}

In this section we would prove a suitable Caccioppoli inequality. First of all we recall two useful inequalities. The first is the Sobolev embedding theorem which yields the existence of a constant $C_{s}$ depending only on $s, n$, and $N$ such that for $x_{0} \in D, \rho \leq 1-\left|x_{0}\right|$ there holds

$$
\sup _{B_{\rho}^{+}\left(x_{0}\right)}\left|g-g_{x_{0}, \rho}^{\prime}\right| \leq C_{s} \rho^{1-(n / s)}\|g\|_{H^{1, s}\left(B_{\rho}^{+}\left(x_{0}\right), R^{N}\right)} .
$$

Obviously, the inequality remains true if we replace $\|g\|_{H^{1, s}\left(B_{\rho}^{+}\left(x_{0}\right), R^{N}\right)}$ by $\|g\|_{H^{1, s}\left(B^{+}, R^{N}\right)}$, which we will henceforth abbreviate simply as $\|g\|_{H^{1, s}}$.

Next we note that the Poincaré inequality in this setting for $x_{0} \in D, \rho \leq 1-\left|x_{0}\right|$ yields

$$
\int_{B_{\rho}^{+}\left(x_{0}\right)}\left|g-g_{x_{0}, \rho}\right|^{m} d x \leq C_{p} \rho^{m} \int_{B_{\rho}^{+}\left(x_{0}\right)}|D g|^{m} d x,
$$

for a constant $C_{p}$ which depends only on $n$.

Finally, we fix an exponent $\sigma \in(0,1)$ as follows: if $g \equiv 0$, $\sigma$ can be chosen arbitrarily (but henceforth fixed); otherwise we take $\sigma$ fixed in $(0,1-(n / s))$.

Then we establish an appropriate inequality for Caccioppoli.

Theorem 8 (Caccioppoli's inequality). Let $u \in W^{1, m}(\bar{\Omega}$, $\left.R^{N}\right) \cap L^{\infty}\left(\bar{\Omega}, R^{N}\right)$ with $\|u\|_{L^{\infty}} \leq M<\infty$ and $2 a(M) M<$ $\lambda$ be a weak solution of systems (1) under assumption conditions (H1)-(H5). Then there exists $\rho_{0}\left(L, M, a(M), s,\|g\|_{H^{1, s}}\right)>0$ such that, for all $B_{\rho}^{+}\left(x_{0}\right) \subset B^{+}$, with $x_{0} \in D^{+}, 0<\rho<R<\rho_{0}$, there holds

$$
\begin{aligned}
& \int_{B_{\rho / 2}^{+}\left(x_{0}\right)}|D u|^{2} d x \leq C_{1} \int_{B_{\rho}^{+}\left(x_{0}\right)} \frac{\left|u(x)-u_{x_{0}, R}^{\prime}\right|^{2}}{\rho^{2}} d x+C_{2} \alpha_{n} \rho^{n} \\
& \quad+C_{3}\left(\alpha_{n} \rho^{n}\right)^{1-(2 / s)}\|g\|_{H^{1, s}}^{2},
\end{aligned}
$$

where $C_{1}$ depends only on $\lambda, L$, and $M$ and $C_{3}$ depends on these quantities, and in addition to $C_{p}, C_{2}$ depends on $\lambda, L, M, a, b$, and $\|g\|_{L^{\infty}\left(B, R^{N}\right)}$. 
Proof. Consider a cutoff function $\eta \in C_{0}^{\infty}\left(B_{\rho / 2}^{+}\left(x_{0}\right)\right)$, satisfying $0 \leq \eta \leq 1, \eta \equiv 0$ on $B_{\rho / 2}^{+}\left(x_{0}\right)$ and $|\nabla \eta|<4 / \rho$. Then the function $(u-g) \eta^{2}$ is in $W_{0}^{1, m}\left(B_{\rho / 2}^{+}\left(x_{0}, R^{N}\right)\right)$ and thus can be taken as a test-function.

Using (H1), (H4), (H5), and Young's inequality and noting that $2 a(M) M<\lambda$, we can get from (8) with $\varepsilon$ positive but arbitrary (to be fixed later)

$$
\begin{aligned}
& \int_{B_{\rho}^{+}\left(x_{0}\right)} A_{i j}^{\alpha \beta}(\cdot, u)\left(D_{\beta} u^{j}, D_{\alpha} u^{i}\right) \eta^{2} d x \\
& \leq L \int_{B_{\rho}^{+}\left(x_{0}\right)}\left(1+|u|^{2}\right)^{(m-2) / 2}|D g||D u| \eta^{2} d x \\
& +2 L \int_{B_{\rho}^{+}\left(x_{0}\right)}\left(1+|u|^{2}\right)^{(m-2) / 2}|D \eta||D u| \eta|u-g| d x \\
& +a \int_{B_{\rho}^{+}\left(x_{0}\right)}|D u|^{m}|u-g| \eta^{2} d x+b \int_{B_{\rho}^{+}\left(x_{0}\right)}|u-g| \eta^{2} d x \\
& \leq \varepsilon \int_{B_{\rho}^{+}\left(x_{0}\right)}\left(1+|u|^{2}\right)^{(m-2) / 2}|D u|^{2} \eta^{2} d x \\
& +\underset{B_{\rho}^{+}\left(x_{0}\right)}{a \sup _{0}}\left|u-u_{x_{0}, \rho}^{\prime}\right| \int_{B_{\rho}^{+}\left(x_{0}\right)}|D u|^{m} \eta^{2} d x \\
& +a \sup _{B_{\rho}^{+}\left(x_{0}\right)}\left|g-g_{x_{0}, \rho}^{\prime}\right| \int_{B_{\rho}^{+}\left(x_{0}\right)}|D u|^{m} \eta^{2} d x \\
& +\frac{L^{2}}{2 \varepsilon} \int_{B_{\rho}^{+}\left(x_{0}\right)}\left(1+|u|^{2}\right)^{(m-2) / 2}|D g|^{2} \eta^{2} d x \\
& +\frac{4 L^{2}}{\varepsilon} \int_{B_{\rho}^{+}\left(x_{0}\right)}\left(1+|u|^{2}\right)^{(m-2) / 2}|D \eta|^{2}\left|u-u_{x_{0}, \rho}^{\prime}\right|^{2} d x \\
& +\frac{4 L^{2}}{\varepsilon} \int_{B_{\rho}^{+}\left(x_{0}\right)}\left(1+|u|^{2}\right)^{(m-2) / 2}|D \eta|^{2}\left|g-g_{x_{0}, \rho}^{\prime}\right|^{2} d x \\
& +\frac{\varepsilon}{2} b^{2} \int_{B_{\rho}^{+}\left(x_{0}\right)} \rho^{2} \eta^{2} d x+\frac{1}{\varepsilon \rho^{2}} \int_{B_{\rho}^{+}\left(x_{0}\right)}\left|u-u_{x_{0}, \rho}^{\prime}\right|^{2} d x \\
& +\frac{1}{\varepsilon \rho^{2}} \int_{B_{\rho}^{+}\left(x_{0}\right)}\left|g-g_{x_{0}, \rho}^{\prime}\right|^{2} d x \\
& \leq \varepsilon \int_{B_{\rho}^{+}\left(x_{0}\right)}\left(1+|u|^{2}\right)^{(m-2) / 2}|D u|^{2} \eta^{2} d x \\
& +a\left(M+\|g\|_{L^{\infty}\left(B^{+}, R^{N}\right)}\right) \int_{B_{\rho}^{+}\left(x_{0}\right)}|D u|^{m} \eta^{2} d x \\
& +\frac{64 L^{2}+1}{\varepsilon} \int_{B_{\rho}^{+}\left(x_{0}\right)}\left(1+|u|^{2}\right)^{(m-2) / 2} \frac{1}{\rho^{2}}\left|u-u_{x_{0}, \rho}^{\prime}\right|^{2} d x \\
& +\frac{\varepsilon}{4} b^{2} \eta^{2} \alpha_{n} \rho^{n+2} \\
& +\left(\frac{L^{2}}{2 \varepsilon}+\frac{64 L^{2} C_{p}}{2 \varepsilon}+\frac{4 C_{p}}{\varepsilon}\right)
\end{aligned}
$$

$$
\begin{aligned}
& \cdot \int_{B_{\rho}^{+}\left(x_{0}\right)}\left(1+|u|^{2}\right)^{(m-2) / 2}|D g|^{2} \eta^{2} d x \\
& \leq \varepsilon \int_{B_{\rho}^{+}\left(x_{0}\right)}\left(1+|u|^{2}\right)^{(m-2) / 2}|D g|^{2} \eta^{2} d x \\
& +a\left(M+\|g\|_{L^{\infty}\left(B^{+}, R^{N}\right)}\right) C\left(\|u\|_{W^{1, m}\left(B_{\rho}^{+}\left(x_{0}\right)\right)}\right) \alpha_{n} \rho^{n} \\
& +\frac{64 L^{2}+1}{\varepsilon} \int_{B_{\rho}^{+}\left(x_{0}\right)}\left(1+|u|^{2}\right)^{(m-2) / 2}\left(\frac{u-u_{x_{0}, \rho}^{\prime}}{\rho}\right)^{2} d x \\
& +\frac{\varepsilon}{4} b^{2} \eta^{2} \alpha_{n} \rho^{n+2} \\
& +\left(1+M^{2}\right)^{(m-2) / 2}\left(\frac{L^{2}}{2 \varepsilon}+\frac{64 L^{2} C_{p}}{2 \varepsilon}+\frac{4 C_{p}}{\varepsilon}\right) \\
& \quad \cdot \int_{B_{\rho}^{+}\left(x_{0}\right)}|D g|^{2} \eta^{2} d x .
\end{aligned}
$$

Using (H2), (19), and (20), we thus have

$$
\begin{aligned}
& (\lambda-\varepsilon) \int_{B_{\rho}^{+}\left(x_{0}\right)}|D u|^{2} \eta^{2} d x \\
& \leq(\lambda-\varepsilon) \int_{B_{\rho}^{+}\left(x_{0}\right)}\left(1+|u|^{2}\right)^{(m-2) / 2}|D u|^{2} \eta^{2} d x \\
& \leq \quad \frac{64 L^{2}+1}{\varepsilon} \int_{B_{\rho}^{+}\left(x_{0}\right)}\left(1+|u|^{2}\right)^{(m-2) / 2} \frac{1}{\rho^{2}}\left|u-u_{x_{0}}^{\prime}\right|^{2} d x \\
& \quad+C\left(a, M,\|g\|_{L^{\infty}\left(B^{+}, R^{N}\right)},\|u\|_{W^{1, m}\left(B_{\rho}^{+}\left(x_{0}\right)\right)}, b\right) \alpha_{n} \rho^{n} \\
& \quad+\left(L, C_{p}, M\right) \int_{B_{\rho}^{+}\left(x_{0}\right)}|D g|^{2} d x \\
& \leq \\
& \quad \frac{64 L^{2}+1}{\varepsilon}\left(1+M^{2}\right)^{(m-2) / 2} \int_{B_{\rho}^{+}\left(x_{0}\right)} \frac{1}{\rho^{2}}\left|u-u_{x_{0}}^{\prime}\right|^{2} d x \\
& \quad+C\left(a, M,\|g\|_{L^{\infty}\left(B^{+}, R^{N}\right)}\|u\|_{W^{1, m}\left(B_{\rho}^{+}\left(x_{0}\right)\right)}, b\right) \alpha_{n} \rho^{n} \\
& \quad+\left(L, C_{p}, M\right)\left(\alpha_{n} \rho^{n}\right)^{1-(2 / s)}\|g\|_{H^{1, s}}^{2} .
\end{aligned}
$$

Thus, we fix $\varepsilon$ small enough to yield the desired inequality.

\section{The Proof of the Main Theorem}

In this section we proceed to the proof of the partial regularity result. 
Lemma 9. Consider $u \in W^{1, m}\left(\bar{\Omega}, R^{N}\right) \cap L^{\infty}\left(\bar{\Omega}, R^{N}\right)$ to be a weak solution of (1), $x_{0} \in D$ and $y \in D_{R}\left(x_{0}\right), D_{\rho}(y) \subset \subset$ $D_{R}\left(x_{0}\right)$, for $R<1-\left|x_{0}\right|$, and $\varphi \in C_{0}^{\infty}\left(B_{\rho / 2}^{+}(y), R^{N}\right)$ with $\sup |D \varphi| \leq 1$. We have

$B_{\rho}^{+}(y)$

$$
\begin{gathered}
\left(\frac{\rho}{2}\right)^{2-n} \int_{B_{\rho / 2}^{+}(y)} A_{i j}^{\alpha \beta}\left(y, u_{y, \rho}^{+}\right)\left(D_{\beta} u^{j}, D_{\alpha} \varphi^{i}\right) d x \\
\leq C_{4} \sqrt{I}(\sqrt{I}+\omega(I)) \rho \sup _{B_{\rho / 2}^{+}\left(x_{0}\right)}|D \varphi| .
\end{gathered}
$$

Here and hereafter, we define

$$
I(z, r)=f_{B_{r}^{+}(z)}\left|u-u_{z, r}^{\prime}\right|^{2} d x+\|g\|_{H^{1, s}}^{2} r^{2(1-(n / s))}+r^{2},
$$

for $z \in D, r \in(0,1-|z|)$.

Proof. Using (8) we have

$$
\begin{aligned}
& \int_{B_{\rho / 2}^{+}(y)} A_{i j}^{\alpha \beta}\left(y, u_{y, \rho}^{\prime}\right)\left(D_{\beta} u^{j}, D_{\alpha} \varphi^{i}\right) d x \\
& \leq\left[a \int_{B_{\rho / 2}^{+}(y)}|D u|^{m} d x+2^{-n-1} \alpha_{n} b \rho^{n}\right] \cdot \rho \sup _{B_{\rho / 2}^{+}(y)}|D \varphi| \\
& \quad+\int_{B_{\rho / 2}^{+}(y)}\left|A_{i j}^{\alpha \beta}\left(y, u_{y, \rho}^{\prime}\right)-A_{i j}^{\alpha \beta}(x, u)\right| \\
& \quad \cdot|D u| d x \sup _{B_{\rho / 2}^{+}(y)}|D \varphi| .
\end{aligned}
$$

Applying in turn Young's inequality, (H3), the Caccioppoli inequality (Theorem 8), and Jensen's inequality, we calculate from (26)

$$
\begin{aligned}
& \int_{B_{\rho / 2}^{+}(y)} A_{i j}^{\alpha \beta}\left(y, u_{y, \rho}^{\prime}\right)\left(D_{\beta} u^{j}, D_{\alpha} \varphi^{i}\right) d x \\
& \leq\left[a \int_{B_{\rho / 2}^{+}(y)}|D u|^{m} d x+2^{-n-1} \alpha_{n} b \rho^{n}\right] \cdot \rho \\
& +\left[\int_{B_{\rho / 2}^{+}(y)}\left|A_{i j}^{\alpha \beta}\left(y, u_{y, \rho}^{\prime}\right)-A_{i j}^{\alpha \beta}(x, u)\right|^{1 / 2} d x\right]^{1 / 2} \\
& +\left[\int_{B_{\rho / 2}^{+}(y)}|D u|^{2} d x\right]^{1 / 2}
\end{aligned}
$$

$$
\begin{aligned}
& \leq \frac{\alpha_{n} \rho^{n-1}}{2}\left\{\left(a f_{B_{\rho}^{+}(y)}|D u|^{m} x+2^{-n} b\right) \rho^{2}\right\} \\
& +\alpha_{n} \rho^{n-1} \omega\left(\rho^{m}+M^{m-2} f_{B_{\rho}^{+}(y)}\left|u-u_{y, \rho}^{\prime}\right|^{2} d x\right) \\
& \quad \cdot\left\{C_{1} f_{B_{\rho}^{+}(y)}\left|u-u_{y, \rho}^{\prime}\right|^{2} d x+C_{3}\|g\|_{H^{1, s}}^{2} \rho^{2(1-(n / s))}\right. \\
& \left.\quad+C_{2} \rho^{2}\right\}^{1 / 2} \\
& \leq \frac{\alpha_{n} \rho^{n-1}}{2} C_{5} I+\frac{\alpha_{n} \rho^{n-1}}{2} C_{6} \omega(I) \sqrt{I} \\
& \leq C_{7} \alpha_{n} \rho^{n-1}(I+\omega(I) \sqrt{I}),
\end{aligned}
$$

where $C_{5}=a\|u\|_{W^{1, m}}+b, C_{6}=\max \left\{\sqrt{C_{1}}, \sqrt{C_{2}}, \sqrt{C_{3}}\right\}$, and $C_{7}=(1 / 2)\left(C_{5}+C_{6}\right)$, for $z \in D, r \in(0,1-|z|)$. We introduce the notation

$$
I(z, r)=f_{B_{r}^{+}(z)}\left|u-u_{z, r}^{\prime}\right|^{2} d z+\|g\|_{H^{1, s}}^{2} r^{2(1-(n / s))}+r^{2}
$$

and further write $I$ for $I(y, \rho)$. For arbitrary $\varphi \in C_{0}^{\infty}\left(\Omega, R^{N}\right)$ we thus have, by rescalling,

$$
\begin{aligned}
& \int_{B_{\rho / 2}^{+}(y)} A_{i j}^{\alpha \beta}\left(y, u_{y, \rho}^{\prime}\right)\left(D_{\beta} u^{j}, D_{\alpha} \varphi^{i}\right) d x \\
& \leq C_{7} \alpha_{n} \rho^{n-1} \sqrt{I}(\sqrt{I}+\omega(I)) .
\end{aligned}
$$

Multiplying (29) through by $(\rho / 2)^{2-n}$ yields

$$
\begin{aligned}
& \left|\left(\frac{\rho}{2}\right)^{2-n} \int_{B_{\rho / 2}^{+}(y)} A_{i j}^{\alpha \beta}\left(y, u_{y, \rho}^{\prime}\right)\left(D_{\beta} u^{j}, D_{\alpha} \varphi^{i}\right) d x\right| \\
& \quad \leq C_{4} \sqrt{I}(\sqrt{I}+\omega(I)) \rho \sup _{B_{\rho / 2}^{+}\left(x_{0}\right)}|D \varphi|,
\end{aligned}
$$

for $C_{4}$ defined by $C_{4}=2^{n-3} \alpha_{n} C_{7}$.

Lemma 10. Consider u satisfying the conditions of Theorem 2 and $\sigma$ fixed; then we can find $\delta$ and $s_{0}$ together, with positive constants $C_{8}$ such that the smallness conditions: $0<\omega\left(s_{0}\right) \leq$ $\delta / 2$ and $I\left(x_{0}, R\right) \leq C_{8}^{-1} \min \left\{\delta^{2} / 4, s_{0}\right\}$ together, imply the growth condition

$$
I(y, \theta \rho) \leq \theta^{2 \sigma} I(y, \rho) .
$$


Proof. We now set $w=u-g$, using in turn (H1), Young's inequality, and Hölder's inequality. We have from (30)

$$
\begin{aligned}
& \left|\left(\frac{\rho}{2}\right)^{2-n} \int_{B_{\rho / 2}^{+}(y)} A_{i j}^{\alpha \beta}\left(y, u_{y, \rho}^{\prime}\right)\left(D_{\beta} w^{j}, D_{\alpha} \varphi^{i}\right) d x\right| \\
& \leq\left|\left(\frac{\rho}{2}\right)^{2-n} \int_{B_{\rho / 2}^{+}(y)} A_{i j}^{\alpha \beta}\left(y, u_{y, \rho}^{\prime}\right)\left(D_{\beta} u^{j}, D_{\alpha} \varphi^{i}\right) d x\right| \\
& \quad+\left|\left(\frac{\rho}{2}\right)^{2-n} \int_{B_{\rho / 2}^{+}(y)} A_{i j}^{\alpha \beta}\left(y, u_{y, \rho}^{\prime}\right)\left(D_{\beta} g^{j}, D_{\alpha} \varphi^{i}\right) d x\right| \\
& \leq C_{9} \sqrt{I}[\sqrt{I}+\omega(I)] \rho \sup _{B_{\rho / 2}^{+}\left(x_{0}\right)}|D \varphi|,
\end{aligned}
$$

for $C_{9}=\max \left\{C_{4},\left(\alpha_{n} / 2\right)^{1-(n / s)}\right\}$.

We now set $v=w / \gamma$, for $\gamma=C_{9} \sqrt{I}$. From (32) we then have

$$
\begin{aligned}
& \left|\left(\frac{\rho}{2}\right)^{2-n} \int_{B_{\rho / 2}^{+}(y)} A_{i j}^{\alpha \beta}\left(y, u_{y, \rho}^{\prime}\right)\left(D_{\beta} v^{j}, D_{\alpha} \varphi^{i}\right) d x\right| \\
& \quad \leq(\sqrt{I}+\omega(I)) \rho \sup _{B_{\rho / 2}^{+}\left(x_{0}\right)}|D \varphi|,
\end{aligned}
$$

and from (32) we observe from the definition of $C_{9}$ (recalling also the definition of $\gamma$ )

$$
\left(\frac{\rho}{2}\right)^{2-n} \int_{B_{\rho / 2}^{+}(y)}|D v|^{2} d x<1
$$

Further we note

$$
\left.v\right|_{D_{\rho}(y)}=\left.\frac{1}{\gamma} w\right|_{D_{\rho}(y)}=\left.\frac{1}{\gamma}(u-g)\right|_{D_{\rho}(y)} \equiv 0 .
$$

For $\varepsilon>0$ we take $\delta=\delta(n, N, \lambda, L, \varepsilon)$ to be the corresponding $\delta$ from the A-harmonic approximation lemma. Suppose that we could ensure that the smallness condition

$$
\sqrt{I}+\omega(I) \leq \delta
$$

holds. Then in view of (33), (34), and (35) we would be able to apply Lemma 5 to conclude the existence of a function $h \in H^{1,2}\left(B_{\rho / 2}^{+}(y), R^{N}\right)$ which is $A_{i j}^{\alpha \beta}\left(y, u_{y, \rho}^{\prime}\right)$-harmonic, with $\left.h\right|_{D_{\rho / 2}(y)} \equiv 0$ such that

$$
\begin{aligned}
& \left(\frac{\rho}{2}\right)^{2-n} \int_{B_{\rho / 2}^{+}(y)}|D h|^{2} d x \leq 1, \\
& \left(\frac{\rho}{2}\right)^{-n} \int_{B_{\rho / 2}^{+}(y)}|v-h|^{2} d x \leq \varepsilon .
\end{aligned}
$$

For $\theta \in(0,1 / 4]$ arbitrary (to be fixed later), we have from the Campanato theorem, noting (37) and recalling also that $h(y)=0$,

$$
\sup _{B_{\theta \rho}^{+}(y)}|h|^{2} \leq \theta^{2} \rho^{2} \sup _{B_{\rho / 4}^{+}(y)}|D h|^{2} \leq 4 C_{0} \theta^{2} .
$$

Using (38) and (39) we observe

$$
\begin{aligned}
& (\theta \rho)^{-n} \int_{B_{\theta \rho}^{+}(y)}|v|^{2} d x \\
& \leq 2(\theta \rho)^{-n}\left[\int_{B_{\theta \rho}^{+}(y)}|v-h|^{2} d x+\int_{B_{\theta \rho}^{+}(y)}|h|^{2} d x\right] \\
& \leq 2(\theta \rho)^{-n}\left[\left(\frac{\rho}{2}\right)^{n} \varepsilon+\frac{1}{2} \alpha_{n}(\theta \rho)^{n} \sup _{B_{\theta \rho}^{+}(y)}|h|^{2}\right] \\
& \leq 2^{1-n} \theta^{-n} \varepsilon+4 \alpha_{n} C_{0} \theta^{2},
\end{aligned}
$$

and, hence, on multiplying this through by $\gamma^{2}$, we obtain the estimate

$$
(\theta \rho)^{-n} \int_{B_{\theta \rho}^{+}(y)}|w|^{2} d x \leq C_{9}^{2}\left(2^{1-n} \theta^{-n} \varepsilon+4 \alpha_{n} C_{0} \theta^{2}\right) I .
$$

For the time being, we restrict to the case that $g$ does not vanish identically. Recalling that $w=u-g$, using in turn Poincaré's, Sobolev's, and then Hölder's inequalities, and noting also that $u_{y, \theta \rho}^{\prime}=g_{y, \theta \rho}^{\prime}$, thus from (41) we get

$$
\begin{aligned}
& (\theta \rho)^{-n} \int_{B_{\theta \rho}^{+}(y)}\left|u-\mathrm{u}_{y, \theta \rho}^{\prime}\right|^{2} d x \\
& \leq 2(\theta \rho)^{-n}\left[\int_{B_{\theta \rho}^{+}(y)}|u-g|^{2} d x+\int_{B_{\theta \rho}^{+}(y)}\left|g-g_{y, \theta \rho}^{\prime}\right|^{2} d x\right] \\
& \leq 2 C_{9}^{2}\left(2^{1-n} \theta^{-n} \varepsilon+4 \alpha_{n} C_{0} \theta^{2}\right) I \\
& \quad+2 C_{p}(\theta \rho)^{2-n}\left[\frac{1}{2} \alpha_{n}(\theta \rho)^{n}\right]^{1-(2 / s)}\|g\|_{H^{1, s}}^{2} \\
& \leq C_{10}\left(\theta^{-n} \varepsilon+\theta^{2}\right) I+C_{10} \theta^{2(1-(n / s))} I,
\end{aligned}
$$

for $C_{10}=\max \left\{8 \alpha_{n} C_{0} C_{9}^{2}, 2^{2 / s} C_{p} \alpha_{n}^{1-(2 / s)}\right\}$, and provided $\varepsilon=$ $\theta^{n+2}$, we have

$$
(\theta \rho)^{-n} \int_{B_{\theta \rho}^{+}(y)}\left|u-u_{y, \theta \rho}^{\prime}\right|^{2} d x \leq 3 C_{10} \theta^{2(1-(n / s))} I .
$$

Note that fix $\varepsilon=\theta^{n+2}$, which is also fixed $\delta$. Since $\rho \leq 1$, we see from the definition of $I$

$$
\|g\|_{H^{1, s}}^{2}(\theta \rho)^{2(1-(n / s))} \leq \theta^{2(1-(n / s))} I,
$$

and further

$$
(\theta \rho)^{2} \leq \theta^{2} I
$$

Combining these estimates with (43), we can get

$$
I(y, \theta \rho) \leq 3\left(C_{10}+1\right) \theta^{2(1-(n / s))} I .
$$

Choose $\theta \in(0,1 / 4]$ sufficiently small that there holds: $3\left(C_{10}+1\right) \theta^{2(1-(n / s))} \leq \theta^{2 \sigma}$. 
We can see from (46)

$$
I(y, \theta \rho) \leq \theta^{2 \sigma} I .
$$

We now choose $s_{0}>0$ such that $0<\omega\left(s_{0}\right)<(\delta / 2)$ and define $C_{8}$ by

$$
C_{8}=\max \left\{2^{n-1}, 2 C_{9}^{2}+1,2 C_{s}^{2}+1\right\} .
$$

Suppose that we have

$$
I\left(x_{0}, R\right) \leq C_{8}^{-1} \min \left\{\frac{\delta^{2}}{4}, s_{0}\right\},
$$

for some $R \in\left(0, R_{0}\right]$, where $R_{0}=\min \left\{\sqrt{2 s_{0}}, 1-\left|x_{0}\right|\right\}$.

For any $y \in D_{R / 2}\left(x_{0}\right)$ we use the Sobolev inequality to calculate

$$
\begin{aligned}
& \frac{\alpha_{n} R^{n}}{2^{n+1}}\left|u_{x_{0}, R}^{\prime}-u_{y, R / 2}^{\prime}\right|^{2} \\
& \quad=\int_{B_{R / 2}^{+}}\left|u_{x_{0}, R}^{\prime}-u_{y, R / 2}^{\prime}\right|^{2} d x=\int_{B_{R / 2}^{+}}\left|g_{x_{0}, R}^{\prime}-g_{y, R / 2}^{\prime}\right|^{2} d x \\
& \quad \leq 2 \int_{B_{R / 2}^{+}}\left|g-g_{x_{0}, R}^{\prime}\right|^{2} d x+2 \int_{B_{R / 2}^{+}}\left|g-g_{y, R / 2}^{\prime}\right|^{2} d x \\
& \quad \leq 2 \alpha_{n} C_{s}^{2}\|g\|_{H^{1, s}}^{2} R^{n+2(1-(n / s))} .
\end{aligned}
$$

Then we can calculate

$$
\begin{aligned}
& I\left(y, \frac{1}{2} R\right) \\
& \leq 2^{n-1} f_{B_{R / 2}^{+}(y)}\left|u-u_{x_{0}, R}^{\prime}\right|^{2} d x \\
& \quad+\left(2 C_{s}^{2}+1\right)\|g\|_{H^{1, s}}^{2} R^{2(1-(n / s))}+\frac{1}{4} R^{2} \\
& \leq C_{8} I\left(x_{0}, R\right) .
\end{aligned}
$$

Then we have

$$
\begin{aligned}
& \sqrt{I\left(y, \frac{1}{2} R\right)}+\omega\left(I\left(y, \frac{1}{2} R\right)\right) \\
& \leq \sqrt{C_{8} I\left(x_{0}, R\right)}+\sqrt{\omega\left(C_{8} I\left(x_{0}, R\right)\right)} \\
& \leq \frac{1}{2} \delta+\omega\left(s_{0}\right) \leq \delta,
\end{aligned}
$$

which means that the condition (49) is sufficient to guarantee the smallness condition (37) for $\rho=R / 2$, for all $y \in D_{R / 2}\left(x_{0}\right)$. We can thus conclude that (46) holds in this situation. From (46) we thus have

$$
\begin{aligned}
& \sqrt{I\left(y, \frac{\theta \rho}{2}\right)}+\sqrt{\omega\left(I\left(y, \frac{\theta \rho}{2}\right)\right)} \\
& \leq \sqrt{I\left(\mathrm{y}, \frac{1}{2} R\right)}+\sqrt{\omega\left(I\left(y, \frac{1}{2} R\right)\right)} \leq \delta,
\end{aligned}
$$

meaning that we can apply $(46)$ on $B_{\theta \rho / 2}^{+}(y)$ as well, yielding

$$
I\left(y, \frac{\theta^{2} R}{2}\right) \leq \theta^{4 \sigma} I\left(y, \frac{R}{2}\right),
$$

and inductively

$$
I\left(y, \frac{\theta^{k} R}{2}\right) \leq \theta^{2 k \sigma} I\left(y, \frac{R}{2}\right) .
$$

The next step is to go from a discrete to a continuous version of the decay estimate. Given $\rho \in(0, R / 2]$, we can find $k \in N_{0}$ such that $\theta^{k+1} R / 2<\rho \leq \theta^{k} R / 2$. Firstly we use the Sobolev inequality, to see

$$
\begin{aligned}
& \int_{B_{\rho}^{+}(y)}\left|u_{y, \rho}^{\prime}-u_{y, \theta^{k} R / 2}^{\prime}\right|^{2} d x \\
& \leq 2 \alpha_{n}\left(\frac{1}{2 \theta^{k} R}\right)^{n} C_{s}^{2}\|g\|_{H^{1, s}}^{2}\left(\frac{1}{2 \theta^{k} R}\right)^{2(1-(n / s))},
\end{aligned}
$$

which allows us to deduce

$$
\begin{aligned}
& \int_{B_{\rho}^{+}(y)}\left|u-u_{y, \rho}^{\prime}\right|^{2} d x \\
& \leq 2 \int_{B_{\rho}^{+}(y)}\left|u-u_{y, \theta^{k} R / 2^{\prime}}\right|^{2} d x \\
& \quad+4 \alpha_{n}\left(\frac{1}{2 \theta^{k} R}\right)^{n} C_{s}^{2}\|g\|_{H^{1, s}}^{2}\left(\frac{1}{2 \theta^{k} R}\right)^{2(1-(n / s))},
\end{aligned}
$$

and, hence,

$$
I(y, \rho) \leq C_{11} I\left(y, \frac{\theta^{k} R}{2}\right),
$$

for $C_{11}=8 \theta^{-n} C_{s}^{2}+1$. Combining this with (55) and (51), we have

$$
\begin{aligned}
& I(y, \rho) \\
& \quad \leq C_{11} \theta^{2 k \sigma} I\left(y, \frac{R}{2}\right) \leq C_{8} C_{11} \theta^{-2 \sigma}\left(\frac{2 \rho}{R}\right)^{2 \sigma} I\left(x_{0}, R\right) \\
& \quad \leq C_{8} C_{11}\left(\frac{2}{\theta}\right) I\left(x_{0}, R\right)\left(\frac{\rho}{R}\right)^{2 \sigma},
\end{aligned}
$$

and more particularly

$$
\inf _{\mu \in R^{N}} \int_{B_{\rho}^{+}(y)}|u-\mu|^{2} d x \leq C_{12} I\left(x_{0}, R\right)\left(\frac{\rho}{R}\right)^{2 \sigma},
$$

for $C_{12}=C_{8} C_{11}(2 / \theta)^{2 \sigma}$. Recall that this estimate is valid for all $y \in D$ and $\rho$ with $D_{\rho}(y) \subset D_{R / 2}\left(x_{0}\right)$; assume only the condition (49) on $I\left(x_{0}, R\right)$. This yields after replacing $R$ with $6 R$ the boundary estimate (15) which requires to apply Lemma 6.

Combining the boundary and interior estimates [19] we can derive the desired result. As the argument for combining the boundary and interior regularity results is relatively standard, we omit it. Hence we can apply Lemma 6 and conclude the desired Hölder continuity. 


\section{Acknowledgments}

This work was supported by the National Natural Science Foundation of China (nos. 11201415, 11271305), Natural Science Foundation of Fujian Province (2012J01027), and Training Programme Foundation for Excellent Youth Researching Talents of Fujian's Universities (JA12205).

\section{References}

[1] M. Giaquinta and G. Modica, "Partial regularity of minimizers of quasiconvex integrals," Annales de l'institut Henri Poincaré, vol. 3, pp. 185-208, 1986.

[2] M. Giaquinta, "A counter-example to the boundary regularity of solutions to elliptic quasilinear systems," Manuscripta Mathematica, vol. 24, no. 2, pp. 217-220, 1978.

[3] E. Giusti and M. Miranda, "Sulla regolarità delle soluzioni deboli di una classe di sistemi ellittici quasi-lineari," Archive for Rational Mechanics and Analysis, vol. 31, no. 3, pp. 173-184, 1968.

[4] A. A. Arkhipova, "Regularity results for quasilinear elliptic systems with nonlinear boundary conditions," Journal of Mathematical Sciences, vol. 77, no. 4, pp. 3277-3294, 1995.

[5] A. A. Arkhipova, "On the regularity of solutions of boundaryvalue problem for quasilinear elliptic systems with quadratic nonlinearity," Journal of Mathematical Sciences, vol. 80, no. 6, pp. 2208-2225, 1996.

[6] A. A. Arkhipova, "On the regularity of the oblique derivative problem for quasilinear elliptic systems," Journal of Mathematical Sciences, vol. 84, no. 1, pp. 817-822, 1997.

[7] M. Wiegner, "A-Priori Schranken für Lösungen gewisser elliptischer Systeme," Manuscripta Mathematica, vol. 18, no. 3, pp. 279-297, 1976.

[8] S. Hildebrandt and K. O. Widman, "On the Hölder continuity of weak solutions of quasilinear elliptic systems of second order," Annali della Scuola Normale Superiore di Pisa IV, vol. 4, pp. 144$178,1977$.

[9] J. Jost and M. Meier, "Boundary regularity for minima of certain quadratic functionals," Mathematische Annalen, vol. 262, no. 4, pp. 549-561, 1983.

[10] F. Duzaar, J. Kristensen, and G. Mingione, "The existence of regular boundary points for non-linear elliptic systems: to the memory of Sergio Campanato," Journal fur die Reine und Angewandte Mathematik, vol. 2007, no. 602, pp. 17-58, 2007.

[11] J. Kristensen and G. Mingione, "Boundary regularity in variational problems," Archive for Rational Mechanics and Analysis, vol. 198, no. 2, pp. 369-455, 2010.

[12] S. Hildebrandt, "Nonlinear elliptic systems and harmonic mappings," in Proceedings of the 1980 Beijing Symposium on Differential Geometry and Differential Equations, vol. 1, pp. 481-615, Science Press, Beijing, China, 1982, See also: Vorlesungsreihe 3, SFB 72, Universit Äat, Bonn, Germany, 1980.

[13] S. Hildebrandt, "Quasilinear elliptic systems in diagonal form," in Systems of Nonlinear Partial Differential Equations, J. M. Ball, Ed., pp. 173-217, Proceedings of Nato Advanced Study Institute, Oxford, UK, 1983.

[14] J. F. Grotowski, "Boundary regularity for quasilinear elliptic systems," Communications in Partial Differential Equations, vol. 27, no. 11-12, pp. 2491-2512, 2002.

[15] F. Duzaar and K. Steffen, "Optimal interior and boundary regularity for almost minimal currents to elliptic integrands,"
Journal für die Reine und Angewandte Mathematik, vol. 546, pp. 73-138, 2002.

[16] F. Duzaar and J. F. Grotowski, "Optimal interior partial regularity for nonlinear elliptic systems: the method of A-harmonic approximation," Manuscripta Mathematica, vol. 103, no. 3, pp. 267-298, 2000.

[17] J. F. Grotowski, "Boundary regularity for nonlinear elliptic systems," Calculus of Variations and Partial Differential Equations, vol. 15, no. 3, pp. 353-388, 2002.

[18] W. K. Allard, "On the first variation of a varifold," Annals of Mathematics, vol. 95, pp. 225-254, 1972.

[19] M. Giaquinta, Multiple Integrals in the Calculus of Variations and Nonlinear Elliptic Systems, Princeton University Press, Princeton, NJ, USA, 1983.

[20] S. Campanato, "Equazioni ellittiche del $\mathrm{II}^{\circ}$ ordine e spazi $L^{2, \lambda}$," Annali di Matematica Pura ed Applicata, vol. 69, no. 1, pp. 321381, 1965.

[21] S. Campanato, "Proprietà di Hölderianità di alcune classi di funzioni," Annali della Scuola Normale Superiore di Pisa Series III, vol. 17, pp. 175-188, 1963. 


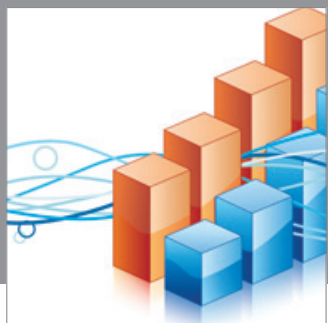

Advances in

Operations Research

mansans

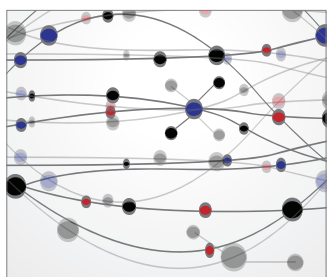

The Scientific World Journal
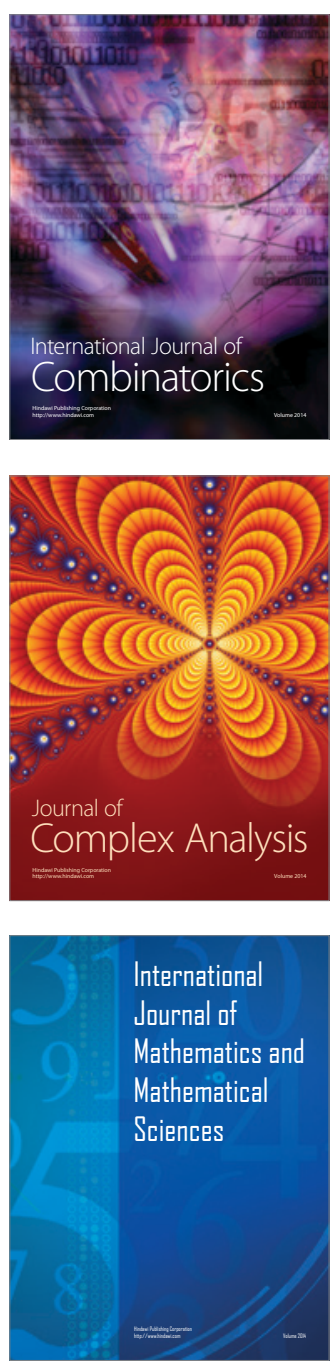
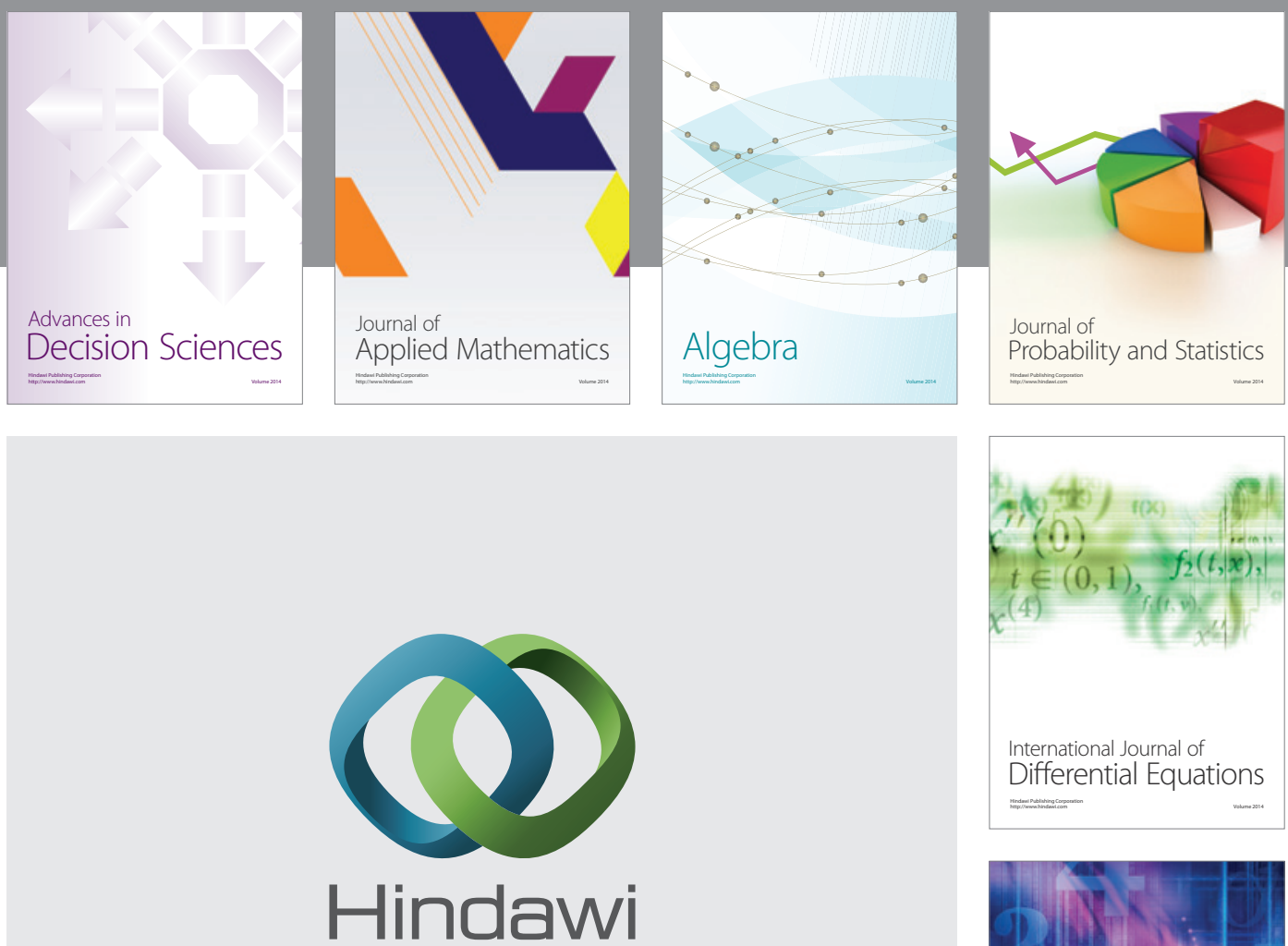

Submit your manuscripts at http://www.hindawi.com
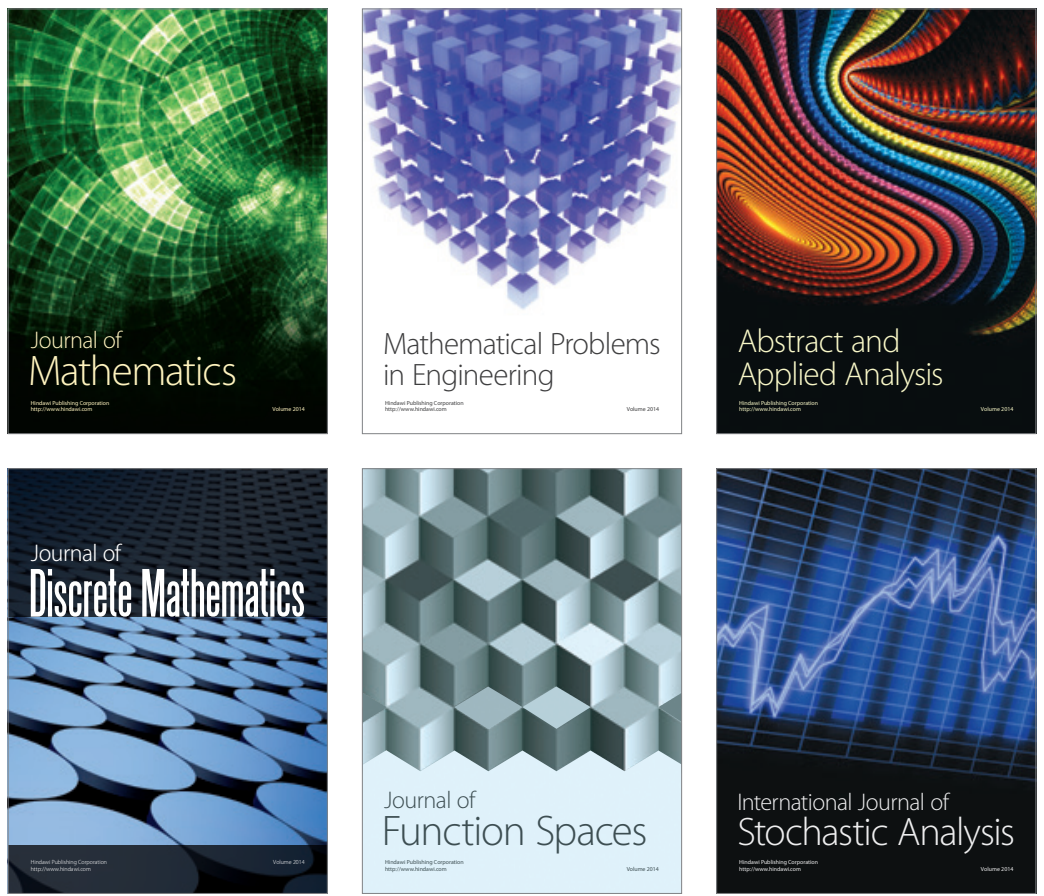

Journal of

Function Spaces

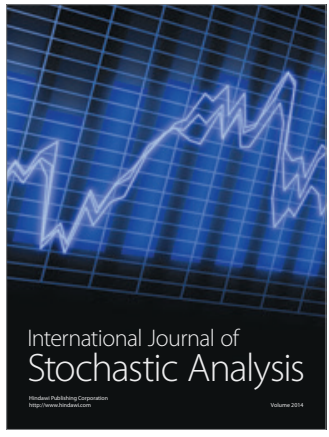

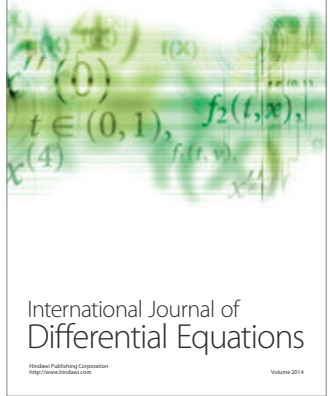
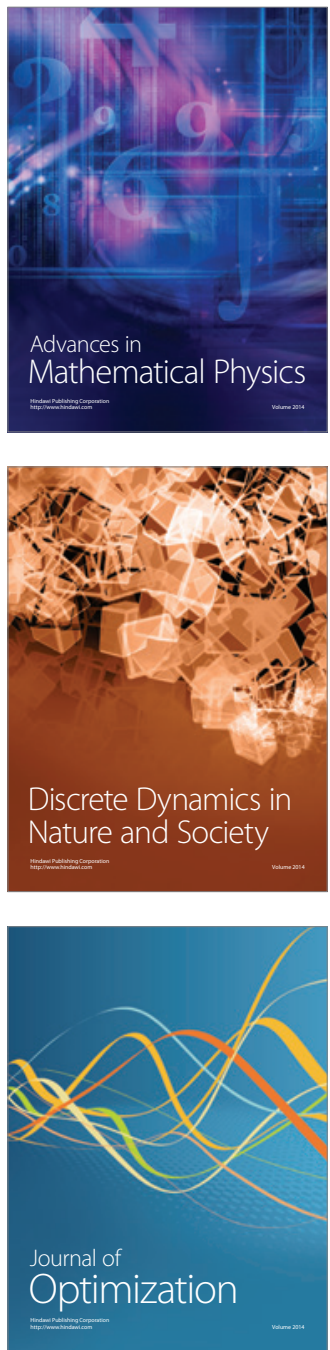